\title{
La formación en religiosidad y espiritualidad en los estudios de Grado en Enfermería
}

\section{The formation in religiosity and spirituality in the studies of Degree in Nursing \\ A formação em religiosidade e espiritualidade nos estudos de Graduação em Enfermagem}

\author{
Rocío de Diego Cordero ${ }^{1}$, Cristina Suero Castillo², Juan Vega \\ Escaño ${ }^{3}$
}

\begin{abstract}
${ }^{1} \mathrm{PhD}, \mathrm{BSc}, \mathrm{OHN}, \mathrm{RN}$. Departamento de Enfermería. Facultad de Enfermería, Fisioterapia y Podología. Universidad de Sevilla. Correo electrónico: rdediego2@us.es

${ }^{2} R N$. Facultad de Enfermería, Fisioterapia y Podología. Universidad de Sevilla. Correo electrónico: cristinasuercastillo@gamil.com

${ }^{3}$ PhD student, MSN, OHN, RN. Centro Universitario de Enfermería Cruz Roja adscrito a la Universidad de Sevilla. Correo electrónico: juanvegadue@gmail.com

Cómo citar este artículo en edición digital: De Diego Cordero, R., Suero Castillo, C. EVega Escaño, J. (2019). La formación en religiosidad y espiritualidad en los estudios de Grado en Enfermería. Cultura de los Cuidados (Edición digital), 23(53). Recuperado de http://dx.doi.org/10.14198/cuid.2019.53.29

Correspondencia: Rocío de Diego Cordero. Departamento de Enfermería. Facultad de Enfermería, Fisioterapia y Podología. Universidad de Sevilla. C/ Avenzoar, n.ํ9, 41003-Sevilla (Spain)

Correo electrónico de contacto: rdediego2@us.es

Recibido: 10/10/2018; Aceptado: 11/01/2019
\end{abstract}

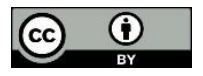

\section{ABSTRACT}

The objective of this investigation has been to investigate the convenience of including religiosity/spirituality in the study plans of the nursing degree. To this end, a review of the scientific literature of the last 5 years (2012-17) has been carried out in the Cinalh, Scopus, Pubmed and Web Of Science (WOS) international databases. The descriptors used were "religion", "spirituality", "student", "nursing" and "education". The results suggest that the training needs of nursing students vary according to the population studied. There are also barriers in the acquisition of these skills in practice with the existence of a materialistic nursing, focused on the biological and/or the fear of being misinterpreted. The bibliography evidences that including the subject of religiosity / spirituality in the nursing study plans obtains positive results in nursing care and in the personal sphere of the students themselves. As analyzed, it is considered convenient to include the subject of religiosity/spirituality in nursing degree studies due to the existing knowledge gap on this subject and the benefits associated with the treatment of these issues.

Keywords: Spirituality, religiosity, nursing students, education, training. 


\section{RESUMO}

O objetivo desta investigação foi investigar a conveniência de incluir religiosidade/espiritualidade nos planos de estudo do grau de enfermagem. Para este fim, uma revisão da literatura científica dos últimos 5 anos (2012-17) foi realizada nos bancos de dados Cinahl, Scopus, Pubmed e Web of Science (WOS). Os descritores usados foram "religião", "espiritualidade", "aluno", "enfermagem" e "educação". Os resultados sugerem que as necessidades de treinamento de estudantes de enfermagem variam de acordo com a população estudada. Há também barreiras na aquisição dessas habilidades na prática com a existência de uma enfermagem materialista, focada no biológico e/ou medo de ser mal interpretada. A bibliografia evidencia que a inclusão do tema da religiosidade/espiritualidade nos planos de estudo em enfermagem obtém resultados positivos no cuidado de enfermagem e na esfera pessoal dos próprios estudantes. Conforme analisado, é considerado conveniente incluir o tema da religiosidade/espiritualidade em estudos de graduação de enfermagem devido à lacuna de conhecimento existente sobre este assunto e aos benefícios associados ao tratamento dessas questões.

Palavras-chave:

Espiritualidade, religiosidade, estudantes de enfermagem, educação, treinamento.

\section{RESUMEN}

El objetivo de esta investigación ha sido indagar la conveniencia de incluir materia de religiosidad/espiritualidad en los planes de estudio del grado en enfermería.

Para ello se ha llevado a cabo una revisión de la literatura científica de los últimos 5 años (2012-2017) en las bases de datos internacionales Cinalh, Scopus, Pubmed y
Web Of Science (WOS). Los descriptores usados fueron "religión", "espiritualidad", "estudiante", "enfermería" y "educación". Los resultados apuntan que las necesidades de formación de los estudiantes de enfermería varían según la población estudiada. También se evidencian barreras en la adquisición de estas competencias en la práctica con la existencia de una enfermería materialista, centrada en lo biológico y/o el temor a ser malinterpretados. La bibliografía evidencia que incluir la materia de religiosidad/espiritualidad en los planes de estudio de enfermería obtiene resultados positivos en los cuidados de enfermería y en la esfera personal del propio alumnado. Según lo analizado, se considera conveniente incluir la materia de religiosidad/espiritualidad en los estudios de grado en enfermería debido a la brecha de conocimientos existente acerca de este tema y los beneficios asociados al tratamiento de estas cuestiones.

\section{Palabras clave: Espiritualidad,} religiosidad, estudiantes de enfermería, educación, formación.

\section{INTRODUCCIÓN}

La cultura es un conjunto de creencias, ritos y acuerdos sobre cómo concebir e interpretar el mundo donde lo religioso/espiritual es un factor central (Aznar, 2016). No obstante, el abordaje de estas cuestiones es escaso en el ámbito de la educación superior. En el caso concreto de la formación en Enfermería, la bibliografía evidencia que tanto profesionales como estudiantes sienten la necesidad de recibir formación acerca de cómo satisfacer las necesidades espirituales de los usuarios a los que atienden (Sanabria Triana, Otero 
Ceballos y Urbina Laza, 2002), no abordándose estas cuestiones desde la enseñanza universitaria.

Proporcionar asistencia religiosa promueve la humanización del sistema sanitario y se traduciría en una asistencia integral del paciente, de acuerdo con la definición de salud que propone la Organización Mundial de la Salud (Ministerio de Sanidad, 2011). Enfermería es una disciplina que se ha interesado por mirar a la persona desde una perspectiva integral y la tendencia debe de ser un aumento del interés por las necesidades espirituales de los/las pacientes. Entre las razones de brindar cuidados espirituales se encuentran los beneficios que se obtiene de esta práctica para el paciente como para el propio profesional: el/la paciente se siente valorado/a, acepta mejor su enfermedad, mantiene esperanzas en su curación y se siente más resistente; el personal de enfermería siente satisfacción de haber brindado cuidados humanizados, obtiene emociones positivas y con ellos aumenta la calidad de la vida laboral. Otra de las razones radica en las limitaciones del saber de la ciencia. Cuando la ciencia ha agotado sus motivos, la espiritualidad/religión puede suponer otras fuente de apoyo para la persona, y para el/la enfermero/a es una potente herramienta que ayuda al paciente en su proceso de salud-enfermedad (Nascimento et al., 2016). De no tener en cuenta las necesidades religiosas/espirituales de los/las usuarios/as éstos/as se mostrarán tensos/as e insatisfechos/as con la asistencia recibida dando lugar a resultados desfavorables, en ocasiones, con consecuencias muy negativas (Melguizo y Alzate, 2008).

La bibliografía muestra una larga lista de beneficios que la religión/espiritualidad ejerce en el proceso de salud-enfermedad.
Sin embargo, en la práctica las necesidades fisiológicas de los/las pacientes son atendidas de manera prioritaria (Nascimento et al., 2016) de forma que las necesidades espirituales no las reconocen, no las satisfacen o no hacen frente a ellas.

Satisfacer las necesidades religiosas de los/las pacientes pasan a no ser una opción sino un componente esencial del cuidado de enfermería. Enfermería queda en el compromiso de ofrecer una atención integral al paciente (Neil Cope, Azofeifa Ugalde y Calderón Montero, 2012); por ello es necesario que los/las futuros/as profesionales de enfermería atiendan a las necesidades espirituales y contemplen al ser humano en toda su complejidad (Melguizo Herrera y Alzate Posada, 2008).

\section{OBJETIVO}

Indagar la conveniencia de incluir materia de religiosidad/espiritualidad en los planes de estudio del grado en enfermería.

\section{METODOLOGÍA}

Se ha realizado una búsqueda de bibliografía científica en las bases de datos Pubmed, Cinahl, Scopus y Web of Science (WOS), seleccionado los trabajos publicados en los últimos 5 años (2012-2017). Los descriptores usados fueron "religión", "espiritualidad", "estudiante", "enfermería" y "educación", combinados mediante los booleanos AND y OR. Los criterios de inclusión fueron artículos con acceso a texto completo, publicados entre 2012-2017, en los idiomas inglés, portugués y español y los criterios de exclusión fueron que estuviesen escritos en idiomas distintos a los indicados, como coreano y chino, así como que se tratase de fuentes repetidas. La búsqueda obtuvo un total de 611 artículos 
potencialmente relevantes de los que se han seleccionado 28 .

\section{RESULTADOS}

Los principales hallazgos identificados tras el análisis de la bibliografía fueron "necesidades de formación en religiosidad/espiritualidad del alumnado de enfermería" (A); "principales dificultades del alumnado de enfermería al abordar temas de religiosidad/espiritualidad" (B) y "beneficios de incluir materia de religiosidad/espiritualidad en los planes de estudios del grado en enfermería" (C), quedando recogidos en la siguiente tabla (Tabla 1):

TABLA 1: Análisis y síntesis de los resultados. Categorización temática

\begin{tabular}{|c|c|c|c|c|c|}
\hline $\begin{array}{c}\text { Autor/es y } \\
\text { año }\end{array}$ & País & $\begin{array}{c}\text { Metodología y } \\
\text { Muestra }\end{array}$ & Objetivos & $\begin{array}{c}\text { Principales } \\
\text { resultados }\end{array}$ & Categoría \\
\hline $\begin{array}{l}\text { (Abbasi et } \\
\text { al., 2014) }\end{array}$ & Irán & $\begin{array}{l}\text { Estudio } \\
\text { descriptivo- } \\
\text { comparativo } \\
\text { ( } 283 \text { estudiantes } \\
\text { de enfermería) }\end{array}$ & $\begin{array}{lr}\text { Comparar } & 3 \\
\text { variables entre los } & \text { los } \\
\text { estudiantes de } \\
\text { enfermería de } 10^{\circ} \text { y } \\
4 .^{\circ} \text { año de carrera: } \\
\text { bienestar espiritual, } \\
\text { espiritualidad y las } \\
\text { perspectivas de } \\
\text { cuidado espiritual }\end{array}$ & $\begin{array}{l}\text { No hubo diferencias } \\
\text { estadísticamente } \\
\text { significativa s entre } \\
\text { los dos grupos en } \\
\text { ninguna de las } \\
\text { variables estudiadas }\end{array}$ & $\mathrm{C}$ \\
\hline $\begin{array}{l}\text { (Burkhart y } \\
\text { Schmidt, } \\
\text { 2012) }\end{array}$ & $\begin{array}{l}\text { Estados } \\
\text { Unidos }\end{array}$ & $\begin{array}{l}\text { Ensayo aleatorio } \\
\text { controlado } \\
\text { ( } 59 \text { estudiantes de } \\
\text { enfermería) }\end{array}$ & $\begin{array}{l}\text { Probar la eficacia de } \\
\text { un programa } \\
\text { educativo } \\
\text { reflexivo } \\
\text { atención espiritual }\end{array}$ & $\begin{array}{l}\text { Se revela un } \\
\text { aumento } \\
\text { estadísticamente } \\
\text { significativo en la } \\
\text { capacidad percibida } \\
\text { de los estudiantes } \\
\text { para proporcionar } \\
\text { cuidado espiritual } \\
\text { además de mejoría } \\
\text { bienestar espiritual } \\
\text { del propio } \\
\text { estudiante }\end{array}$ & $\mathrm{C}$ \\
\hline $\begin{array}{l}\text { (Caldeira et } \\
\text { al., 2016) }\end{array}$ & $\begin{array}{l}\text { Portugal } \\
\text { y Brasil }\end{array}$ & $\begin{array}{l}\text { Estudio } \\
\text { descriptivo } \\
\text { transversal } \\
\text { (129 estudiantes } \\
\text { de enfermería) }\end{array}$ & $\begin{array}{lrr}\text { Estudiar el } & \text { estado } \\
\text { actual de } & \text { la } \\
\text { educación } & \text { en } \\
\text { enfermería } & \text { sobre } \\
\text { espiritualidad } & \end{array}$ & $\begin{array}{lr}\text { Los estudiantes } \\
\text { subrayaron } \\
\text { ausencia } \\
\text { educación da } \\
\text { espiritualidad en el } \\
\text { plan de estudio de } \\
\text { enfermería }\end{array}$ & A \\
\hline $\begin{array}{l}\text { (Cone } \quad \text { y } \\
\text { Giske, } \\
\text { 2013) }\end{array}$ & Noruega & $\begin{array}{l}\text { Estudio } \\
\text { cualitativo } \\
\text { ( } 19 \text { profesores de } \\
\text { enfermería) }\end{array}$ & $\begin{array}{lr}\text { Explorar } & \text { cómo } \\
\text { preparan } & \text { a } \\
\text { estudiantes } & \text { para } \\
\text { reconocer } & \text { las } \\
\text { señales espirituales }\end{array}$ & $\begin{array}{l}\text { Las competencias } \\
\text { aumentan cuando se } \\
\text { les permite evaluar } \\
\text { y proveer cuidados } \\
\text { espirituales en la } \\
\text { práctica }\end{array}$ & $\mathrm{C}$ \\
\hline $\begin{array}{l}\text { (Connors, } \\
\text { Good y } \\
\text { Gollery, } \\
\text { 2017) }\end{array}$ & $\begin{array}{l}\text { Florida } \\
\text { (Estados } \\
\text { Unidos) }\end{array}$ & $\begin{array}{l}\text { Estudio } \\
\text { descriptivo } \\
\text { longitudinal } \\
\text { (26 estudiantes de } \\
\text { enfermería) }\end{array}$ & $\begin{array}{l}\text { Examinar la eficacia } \\
\text { de } 3 \text { estrategias de } \\
\text { enseñanza en } \\
\text { espiritualidad: } \\
\text { educación en el aula, } \\
\text { valoración espiritual } \\
\text { en la práctica y } \\
\text { simulación en el } \\
\text { aula }\end{array}$ & $\begin{array}{l}\text { El impacto de estos } \\
\text { métodos des } \\
\text { enseñanza en las } \\
\text { percepciones de los } \\
\text { estudiantes sobre la } \\
\text { competencia r y } \\
\text { confianza para } \\
\text { abordar aspectos } \\
\text { espirituales de la } \\
\text { atención enfermera } \\
\text { fue } \\
\text { estadísticamente } \\
\text { significativo en las } \\
3 \text { fases del estudio }\end{array}$ & $\mathrm{C}$ \\
\hline
\end{tabular}




\begin{tabular}{|c|c|c|c|c|c|}
\hline $\begin{array}{l}\text { (Cooper y } \\
\text { Chang, } \\
\text { 2016) }\end{array}$ & Australia & $\begin{array}{l}\text { Estudio } \\
\text { cualitativo } \\
(6 \text { estudiantes de } \\
\text { enfermería })\end{array}$ & $\begin{array}{ll}\text { Ver el impacto de } \\
\text { una asignatura } \\
\text { dedicada a cuidados } \\
\text { espirituales } \\
\text { estudiantes } \\
\text { enfermería }\end{array}$ & $\begin{array}{lr}\text { Se muestra la } \\
\text { necesidad de mayor } \\
\text { preparación para } \\
\text { proveer cuidados } \\
\text { espirituales } \\
\text { reconocer mejor la } \\
\text { necesidad } \\
\text { derivar de } \\
\text { profesionales } \\
\text { religiosos }\end{array}$ & $\mathrm{C}$ \\
\hline $\begin{array}{l}\text { (Cooper et } \\
\text { al., 2013) }\end{array}$ & Australia & $\begin{array}{l}\text { Revisión } \\
\text { bibliográfica }\end{array}$ & $\begin{array}{lr}\text { Explorar la } & \text { la } \\
\text { educación del } \\
\text { cuidado espiritual en } \\
\text { los programas de } \\
\text { enfermería }\end{array}$ & $\begin{array}{l}\text { Se evidencian } \\
\text { mejoras en la } \\
\text { percepción propia, } \\
\text { conocimientos y } \\
\text { actitudes, capacidad } \\
\text { para responder } \\
\text { verbalmente al } \\
\text { dolor espiritual }\end{array}$ & $\mathrm{C}$ \\
\hline $\begin{array}{l}\text { (Coscrato y } \\
\text { Villela } \\
\text { Bueno, } \\
2015)\end{array}$ & Brasil & $\begin{array}{l}\text { Estudio } \\
\text { Cualitativo, } \\
\text { utilizando el } \\
\text { método } \\
\text { investigación- } \\
\text { acción } \\
\text { (9 estudiantes de } \\
\text { enfermería) }\end{array}$ & $\begin{array}{l}\text { Indagar las } \\
\text { concepciones de los } \\
\text { estudiantes sobre } \\
\text { espiritualidad para } \\
\text { instaurar acciones } \\
\text { educativas en esta } \\
\text { materia }\end{array}$ & $\begin{array}{l}\text { La educación } \\
\text { académica de } \\
\text { enfermería es un } \\
\text { momento idóneo } \\
\text { para incluir temas } \\
\text { de espiritualidad y } \\
\text { humanización }\end{array}$ & $\mathrm{C}$ \\
\hline $\begin{array}{l}\text { (Costello, } \\
\text { Atinaja- } \\
\text { Faller } \quad \text { y } \\
\text { Hedberg, } \\
\text { 2012) }\end{array}$ & $\begin{array}{l}\text { Simmons } \\
\text { (Estados } \\
\text { Unidos) }\end{array}$ & $\begin{array}{l}\text { Estudio } \\
\text { descriptivo } \\
\text { transversal } \\
\text { (52 estudiantes de } \\
\text { enfermería) }\end{array}$ & $\begin{array}{lr}\text { Saber si la } \\
\text { simulación es un } \\
\text { método eficaz para } \\
\text { instruir } & \text { en } \\
\text { espiritualidad } & \end{array}$ & $\begin{array}{l}\text { Tras la simulación } \\
\text { hubo actitudes más } \\
\text { positivas hacia la } \\
\text { valoración de las } \\
\text { necesidades } \\
\text { espirituales. Esto } \\
\text { les ayudaría a estar } \\
\text { en mayor sintonía } \\
\text { con sus pacientes }\end{array}$ & $\mathrm{C}$ \\
\hline $\begin{array}{l}\text { (Cruz et al., } \\
\text { 2017). }\end{array}$ & $\begin{array}{l}\text { Arabia } \\
\text { Saudí. }\end{array}$ & $\begin{array}{l}\text { Estudio } \\
\text { descriptivo } \\
\text { transversal } \\
\text { (338 estudiantes } \\
\text { de enfermería) }\end{array}$ & $\begin{array}{l}\text { Investigar la } \\
\text { percepción de los } \\
\text { estudiantes sobre el } \\
\text { cuidado espiritual }\end{array}$ & $\begin{array}{l}\text { No entienden la } \\
\text { diferencia entre } \\
\text { cuidado espiritual y } \\
\text { religioso }\end{array}$ & B \\
\hline $\begin{array}{l}\text { (Culatto y } \\
\text { Summerton, } \\
\text { 2015) }\end{array}$ & $\begin{array}{l}\text { Reino } \\
\text { unido }\end{array}$ & $\begin{array}{lr}\text { Estudio } & \\
\text { descriptivo } & \\
\text { transversal } & \\
\text { (59 profesores } & \text { de } \\
\text { estudios } & \text { de } \\
\text { medina } & y \\
\text { enfermería) } & \\
\end{array}$ & $\begin{array}{lr}\text { Conocer cómo las } \\
\text { facultades } & \text { de } \\
\text { medicina } & y \\
\text { enfermería abordan } \\
\text { los cuidados } \\
\text { espirituales. }\end{array}$ & $\begin{array}{l}\text { El } 39,2 \% \text { consideró } \\
\text { que no tenía } \\
\text { personal entrenado } \\
\text { para enseñar a los } \\
\text { alumnos acerca de } \\
\text { espiritualidad. }\end{array}$ & B \\
\hline $\begin{array}{l}\text { (de } \\
\text { Magalhães } \\
\text { Oliveira et } \\
\text { al., 2012) }\end{array}$ & Brasil & $\begin{array}{l}\text { Estudio } \\
\text { cualitativo } \\
(9 \text { estudiantes de } \\
\text { enfermería })\end{array}$ & $\begin{array}{l}\text { Investigar la opinión } \\
\text { de los estudiantes } \\
\text { sobre la dimensión } \\
\text { espiritual del cuidar } \\
\text { en la práctica de } \\
\text { enfermería }\end{array}$ & $\begin{array}{l}\text { La inseguridad es la } \\
\text { principal barrera } \\
\text { para el abordaje de } \\
\text { la dimensión } \\
\text { espiritual según los } \\
\text { participantes }\end{array}$ & B \\
\hline $\begin{array}{l}\text { (Giske, } \\
\text { 2012) }\end{array}$ & Noruega & $\begin{array}{l}\text { Revisión } \\
\text { bibliográfica }\end{array}$ & $\begin{array}{lr}\text { Conocer } & \text { cómo } \\
\text { aprenden } & \text { los } \\
\text { estudiantes } & \text { de } \\
\text { enfermería a brindar } \\
\text { cuidados } \\
\text { espirituales } & \\
\end{array}$ & $\begin{array}{l}\text { Los estudiantes } \\
\text { necesitan ver la } \\
\text { implementación de } \\
\text { la espiritualidad en } \\
\text { la práctica }\end{array}$ & $\mathrm{A}$ \\
\hline $\begin{array}{l}\text { (Giske y } \\
\text { Cone, 2012) }\end{array}$ & Noruega & $\begin{array}{l}\text { Estudio } \\
\text { cualitativo } \\
\text { ( } 42 \text { estudiantes de } \\
\text { enfermería) }\end{array}$ & $\begin{array}{lll}\text { Conocer el } & \text { abordaje } \\
\text { de los } & \text { cuidados } \\
\text { espirituales } & \text { en } & \text { el } \\
\text { alumnado } & & \text { de } \\
\text { enfermería } & & \end{array}$ & $\begin{array}{l}\text { Se evidencia una } \\
\text { falta de abordaje a } \\
\text { la dimensión } \\
\text { espiritual }\end{array}$ & B \\
\hline
\end{tabular}




\begin{tabular}{|c|c|c|c|c|c|}
\hline $\begin{array}{l}\text { (Hsiao et } \\
\text { al., 2012). }\end{array}$ & Taiwán & $\begin{array}{l}\text { Estudio } \\
\text { cuasiexperimental } \\
\text { con estudiantes de } \\
\text { enfermería } \\
\text { (Grupo } \\
\text { experimental } \\
\text { N=39; Grupo } \\
\text { control N=46) }\end{array}$ & $\begin{array}{l}\text { Examinar } \\
\text { efectos de los } \\
\text { programa } \\
\text { aprendizaje } \\
\text { espiritual sobre la } \\
\text { salud mental y el } \\
\text { estrés percibido por } \\
\text { los estudiantes }\end{array}$ & $\begin{array}{l}\text { Mejora la propia } \\
\text { salud espiritual y } \\
\text { alivia el estrés en } \\
\text { los estudiantes de } \\
\text { enfermería }\end{array}$ & $\mathrm{C}$ \\
\hline $\begin{array}{l}\text { (Kalkim, } \\
\text { Midilli } \\
\text { Baysal, } \\
\text { 2016) }\end{array}$ & Turquía & $\begin{array}{l}\text { Estudio } \\
\text { descriptivo } \\
\text { transversal } \\
\text { (400 estudiantes } \\
\text { de enfermería) }\end{array}$ & $\begin{array}{l}\text { Determinar } \\
\text { conocimientos } \\
\text { prácticas y } \\
\text { percepciones de los } \\
\text { estudiantes respecto } \\
\text { a la atención } \\
\text { espiritual. }\end{array}$ & $\begin{array}{l}\text { Se señala como } \\
\text { principales factores } \\
\text { la falta de tiempo, } \\
\text { de conocimiento, } \\
\text { una adecuada } \\
\text { educación, } \\
\text { prioridad a los } \\
\text { problemas físicos }\end{array}$ & B \\
\hline $\begin{array}{l}\text { (Lewinson, } \\
\text { McSherry y } \\
\text { Kevern, } \\
\text { 2015) }\end{array}$ & $\begin{array}{l}\text { Reino } \\
\text { Unido }\end{array}$ & $\begin{array}{l}\text { Revisión } \\
\text { sistemática }\end{array}$ & $\begin{array}{l}\text { Comprender la } \\
\text { posición de la } \\
\text { espiritualidad dentro } \\
\text { de la educación } \\
\text { enfermera }\end{array}$ & 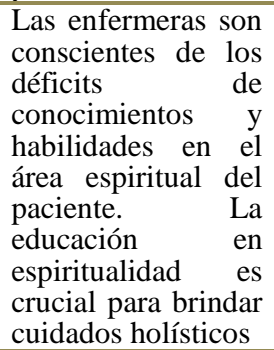 & A \\
\hline $\begin{array}{l}\text { (Murphy } e t \\
a l ., 2015)\end{array}$ & Irlanda & $\begin{array}{l}\text { Estudio } \\
\text { descriptivo } \\
\text { transversal } \\
(543 \text { libros de } \\
\text { enfermería básica) }\end{array}$ & $\begin{array}{lrr}\text { Explorar } & \text { si } & \text { la } \\
\text { espiritualidad } & \text { está } \\
\text { definida en la } \\
\text { literatura científica } \\
\text { sobre enfermería } \\
\text { pediátrica }\end{array}$ & $\begin{array}{l}413(76 \%) \text { libros sin } \\
\text { referencia a la } \\
\text { espiritualidad } \\
\text { términos } \\
\text { relacionados }\end{array}$ & B \\
\hline $\begin{array}{l}\text { (Ross et al., } \\
\text { 2016) }\end{array}$ & $\begin{array}{l}\text { Gales, } \\
\text { Malta, } \\
\text { Noruega } \\
\text { y Países } \\
\text { Bajos }\end{array}$ & $\begin{array}{l}\text { Estudio } \\
\text { descriptivo } \\
\text { transversal } \\
\text { (618 estudiantes } \\
\text { de enfermería) }\end{array}$ & $\begin{array}{lc}\text { Describir } & \text { la } \\
\text { autopercepción } & \text { de } \\
\text { los estudiantes al } \\
\text { brindar espirituales }\end{array}$ & $\begin{array}{lr}\text { Los encuestados } \\
\text { sienten menos } \\
\text { competencia } \\
\text { habilidades en la } \\
\text { aplicación de la } \\
\text { espiritualidad con } \\
\text { calidad }\end{array}$ & B \\
\hline $\begin{array}{l}\text { (Seylani et } \\
\text { al., 2016). }\end{array}$ & Irán & $\begin{array}{l}\text { Estudio } \\
\text { cualitativo } \\
\text { ( } 19 \text { estudiantes de } \\
\text { enfermería) }\end{array}$ & $\begin{array}{l}\text { Explorar el proceso } \\
\text { de desarrollo de la } \\
\text { espiritualidad en los } \\
\text { estudiantes de } \\
\text { enfermería }\end{array}$ & $\begin{array}{lr}\text { El proceso } & \text { de } \\
\text { desarrollo } & \text { de } \\
\text { habilidades } & \\
\text { espirituales } & \text { del } \\
\text { estudiante es } \\
\text { intuitivo y ocurre } \\
\text { principalmente en } \\
\text { el entorno clínico }\end{array}$ & A \\
\hline $\begin{array}{l}\text { (Tiew y } \\
\text { Drury, } \\
\text { 2012) }\end{array}$ & Singapur & $\begin{array}{l}\text { Estudio } \\
\text { cualitativo } \\
\text { (16 estudiantes } \\
\text { enfermería) }\end{array}$ & $\begin{array}{l}\text { Investigar } \quad \text { las } \\
\text { actitudes de los } \\
\text { estudiantes sobre el } \\
\text { cuidado espiritual en } \\
\text { la práctica clínica }\end{array}$ & $\begin{array}{l}\text { Entre los factores } \\
\text { influyentes en la } \\
\text { práctica } \\
\text { entrevistados los } \\
\text { destacan: } \\
\text { enfoque técnico, } \\
\text { falta de tiempo, } \\
\begin{array}{l}\text { diferencia } \\
\text { creencias, el miedo }\end{array}\end{array}$ & B \\
\hline $\begin{array}{l}\text { (Tiew, } \\
\text { Creedy y } \\
\text { Chan, 2013) }\end{array}$ & Singapur & $\begin{array}{l}\text { Estudio descriptivo } \\
\text { transversal } \\
\text { (16 estudiantes de } \\
\text { enfermería de } 3 \\
\text { instituciones } \\
\text { diferentes en el } \\
\text { último año de } \\
\text { grado) }\end{array}$ & $\begin{array}{lr}\text { Investigar } & \text { las } \\
\text { percepciones } & \text { del } \\
\text { cuidado espiritual }\end{array}$ & $\begin{array}{l}\text { La práctica de } \\
\text { enfermería } \\
\text { orientadas al modelo } \\
\text { biomédico dificulta } \\
\text { la comprensión de la } \\
\text { dimensión espiritual } \\
\text { en la práctica }\end{array}$ & B \\
\hline
\end{tabular}




\begin{tabular}{|c|c|c|c|c|c|}
\hline $\begin{array}{l}\text { (Timmins y } \\
\text { Neill, 2013) }\end{array}$ & Irlanda & $\begin{array}{l}\text { Revisión } \\
\text { sistemática }\end{array}$ & $\begin{array}{lr}\text { Examinar } & \text { los } \\
\text { programas para } & \text { la } \\
\text { enseñanza de } & \text { la } \\
\text { espiritualidad } & \text { en } \\
\text { estudiantes } & \text { de } \\
\text { enfermería } & \end{array}$ & $\begin{array}{lr}\text { Los estudiantes } \\
\text { relatan una falta de } \\
\text { información } & \text { sobre } \\
\text { cómo se } & \text { debe } \\
\text { valorar } & \text { la } \\
\text { espiritualidad } & \end{array}$ & B \\
\hline $\begin{array}{l}\text { (Tirgari et } \\
\text { al., 2013) }\end{array}$ & Irán & $\begin{array}{l}\text { Estudio } \\
\text { cualitativo } \\
\text { (11 enfermeras) }\end{array}$ & $\begin{array}{l}\text { Conocer el } \\
\text { significado de los } \\
\text { cuidados } \\
\text { espirituales desde la } \\
\text { propia experiencia } \\
\text { de las enfermeras }\end{array}$ & $\begin{array}{l}\text { Se relata poca } \\
\text { importancia a a } \\
\text { cuidado espiritual } \\
\text { en la práctica }\end{array}$ & B \\
\hline $\begin{array}{l}\text { (Williams et } \\
\text { al., 2016) }\end{array}$ & $\begin{array}{l}\text { Quincy } \\
\text { (Estados } \\
\text { Unidos) }\end{array}$ & $\begin{array}{l}\text { Estudio pre- } \\
\text { experimental } \\
\text { (31 estudiantes de } \\
\text { enfermería) }\end{array}$ & $\begin{array}{l}\text { Examinar el efecto } \\
\text { de la herramienta de } \\
\text { valoración espiritual } \\
\text { FICA sobre la } \\
\text { comodidad } \\
\text { espiritual en el } \\
\text { cuidado del paciente }\end{array}$ & $\begin{array}{l}\text { No hay cambios } \\
\text { estadísticamente } \\
\text { significativos en la } \\
\text { provisión } \\
\text { cuidados de } \\
\text { espirituales tras el } \\
\begin{array}{ll}\text { uso de la } \\
\text { herramienta }\end{array} \\
\end{array}$ & C \\
\hline $\begin{array}{l}\text { (Wu, Liao y } \\
\text { Yeh, 2012) }\end{array}$ & Taiwán & $\begin{array}{l}\text { Estudio } \\
\text { descriptivo } \\
\text { transversal en } \\
(\mathrm{N}=239 \\
\text { estudiantes de } \\
\text { enfermería })\end{array}$ & $\begin{array}{lr}\text { Explorar } & \text { las } \\
\text { percepciones } & \text { del } \\
\text { estudiante acerca del } \\
\text { cuidado espiritual }\end{array}$ & $\begin{array}{l}\text { La educación, la } \\
\text { experiencia y el } \\
\text { interés profesional } \\
\text { afectan a las } \\
\text { percepciones de los } \\
\text { estudiantes de } \\
\text { enfermería sobre la } \\
\text { espiritualidad y el } \\
\text { cuidado espiritual }\end{array}$ & B \\
\hline $\begin{array}{l}\text { (Yilmaz y } \\
\text { Gurler, } \\
\text { 2014) }\end{array}$ & Turquía & $\begin{array}{l}\text { Estudio cuasi- } \\
\text { experimental } \\
(130 \text { estudiantes } \\
\text { de enfermería) }\end{array}$ & \begin{tabular}{lr} 
Medir eficacia & de \\
integrar & \\
espiritualidad & en \\
grado de enfermería & respecto \\
conocimiento & al \\
espiritualidad & y \\
\multicolumn{2}{c}{ cuidado espiritual }
\end{tabular} & $\begin{array}{l}\text { Incrementar } \\
\text { formación en } \\
\text { espiritualidad puede } \\
\text { ser básico para que } \\
\text { se deje de ignorar el } \\
\text { patrón de } \\
\text { espiritualidad de la } \\
\text { valoración } \\
\text { enfermera }\end{array}$ & $\mathrm{C}$ \\
\hline $\begin{array}{l}\text { (Zakaria } \\
\text { Kiaei et al., } \\
\text { 2015) }\end{array}$ & Irán & $\begin{array}{l}\text { Estudio } \\
\text { descriptivo } \\
\text { transversal } \\
(259 \text { enfermeras })\end{array}$ & $\begin{array}{lr}\text { Explorar } & \text { la } \\
\text { percepción de las } \\
\text { enfermeras } \\
\text { relación con la } \\
\text { atención espiritual }\end{array}$ & $\begin{array}{l}\text { Se perciben como } \\
\text { principales } \\
\text { obstáculos los } \\
\text { horarios de trabajo, } \\
\text { estrés laboral y falta } \\
\text { de recursos }\end{array}$ & B \\
\hline
\end{tabular}

FUENTE: Elaboración propia

\section{DISCUSIÓN}

Según los diferentes estudios llevados a cabo en distintas regiones del mundo el alumnado de enfermería necesita contemplar los cuidados espirituales en la práctica y resalta la importancia de que la enseñanza universitaria lo apoye en este ámbito (Giske, 2012); desean conocer qué y cómo valorar el patrón de espiritualidad (Timmins y Neill, 2013) e incluso tienen grandes deseos de sentirse enfermeros y enfermeras competentes y brindar cuidados espirituales con calidad (Cruz et al., 2017).

Respecto a las necesidades de formación en religiosidad/espiritualidad del alumnado de enfermería, según los resultados de Giske (2012) en Noruega, los estudiantes necesitan que los cuidados espirituales se traduzcan a la práctica para aprender además del concepto de espiritualidad, su implementación en la realidad, al igual que aprenden otras habilidades (Giske, 2012). En 
Arabia Saudí, se evidencia la misma necesidad que siente el alumnado noruego, aunque éste tiene una base más pobre sobre conceptos relacionados con religiosidad/espiritualidad ya que no entiende la diferencia entre cuidado religioso y espiritual (Cruz et al., 2017). De igual forma los resultados de un reciente estudio con alumnado iraní muestran que el desarrollo espiritual en el grado de enfermería es intuitivo, llegando a describir su primer año de carrera como frustrante (Seylani et al., 2016). En Irlanda, los/las estudiantes necesitan saber qué y cómo se ha de valorar la espiritualidad ya que desde el ámbito académico se contempla la espiritualidad, pero no se enseña a valorarla en el proceso enfermero (Timmins y Neill, 2013). En un estudio europeo llevado a cabo en 2014 a partir de cuestionarios administrados a 618 estudiantes de enfermería de 6 universidades distintas se pone en evidencia que el alumnado tiene alguna base sobre espiritualidad, pero no se siente competente en la aplicación de cuidados espirituales de calidad (Ross et al., 2016). También en 2016 se llevó a cabo un estudio en Estados Unidos con estudiantes de enfermería a los que se le proporcionó la herramienta de evaluación espiritual (FICA) durante sus prácticas clínicas para que la aplicaran con los pacientes. Ésta no sirvió más que para actuar de guía de valoración, pues los/las estudiantes no mejoraron su capacidad de brindar cuidados espirituales, ni se sintieron más competentes con esta herramienta pues se hacían evidentes sentimientos como incomodidad $\mathrm{O}$ incertidumbre al abordar la espiritualidad. Es por esto que se sugiere que desde el ámbito educativo universitario el alumnado trabaje la autoconciencia espiritual y habilidades de comunicación en contextos espirituales (Williams et al., 2016). Esto último es congruente con la llamada de atención que hacen Giske y Cone (2012) para que desde el ámbito universitario se apoye a los/las estudiantes en su trabajo de autoconciencia espiritual (mediante juegos de rol, estudios de casos) con el fin de detectar y saber responder a las señales espirituales en la práctica.

En lo que respecta a las principales dificultades del alumnado de enfermería en temas de religiosidad/espiritualidad, los estudios apuntan en primer lugar a una falta de importancia a la materia de religiosidad/espiritualidad ya que en la práctica observan que los/las profesionales olvidan estos conceptos y por tanto no pueden adquirir competencias acerca de los cuidados espirituales a pesar de estar motivados para ello (Wu, Liao y Yeh, 2012). En una intervención llevada a cabo por investigadores brasileños con estudiantes de enfermería se señala que la principal dificultad de los cuidados espirituales es la inseguridad, motivada por una falta de conocimiento en este ámbito; aseguran que las competencias en espiritualidad no se adquieren en el grado, por lo que es una importante brecha para el futuro profesional (de Magalhães Oliveira et al., 2012). Las prácticas de enfermería orientadas a las tareas son vistas como un factor que dificulta la comprensión de la espiritualidad en la práctica (Tiew, Creedy y Chan, 2013). Coincide con la percepción del alumnado de Singapur, que además del enfoque materialista, comentan la diferencia de creencias enfermera-paciente y confiesan el miedo a ser malinterpretados (Tiew y Drury, 2012). El enfoque materialista de la enfermería puede tener importante relación con el creciente auge de la dimensión biológica en el mundo de la medicina, hecho que ya ha sido criticado en los resultados del estudio brasileño anteriormente citado 
(Caldeira et al., 2016). Por su parte, el miedo a ser malinterpretados o de imponer las propias creencias puede justificar que los/las estudiantes de enfermería obtengan los conocimientos que necesitan de una religión concreta y de bibliografía científica. Esto evidencia la distorsión existente entre los conceptos espiritualidad y religiosidad, la necesidad de que el alumnado conciban estos conceptos bien diferenciados (Tomasso, Beltrame y Lucchetti, 2011) y el desconocimiento de la numerosa evidencia disponible en la literatura científica acerca de la conexión entre religiosidad/espiritualidad y salud física y/o mental (Espinha et al., 2013). No obstante, según evidencia una intervención llevada a cabo con alumnado taiwanés, el factor "conocimientos" no es el único ítem que dificulta la práctica de la espiritualidad, sino que además influye el interés profesional del alumno/a. De esta forma, la práctica del cuidado espiritual difiere según la conciencia e interés profesional y la satisfacción con la elección de carrera $(\mathrm{Wu}$, Liao y Yeh, 2012). Caldeira et al. llevaron a cabo una intervención con estudiantes de enfermería brasileños y portugueses en el que "muy importante" supuso el $62 \%$ de las respuestas sobre la importancia de la espiritualidad en la formación de enfermería (Caldeira et al., 2016). No obstante, en un estudio en Turquía con 400 estudiantes existe mayor importancia atribuida a la formación en espiritualidad del alumnado de enfermería siendo el 83 '3\% el que desea más información sobre los cuidados espirituales (Kalkim, Midilli y Baysal, 2016). Según la evidencia disponible, existen más factores que influyen en los cuidados espirituales además de los mencionados. Según el estudio brasileño que llevó a cabo Lucchetti (2011), el factor de pertenecer a alguna afiliación religiosa se relaciona directamente con el interés por este tema (Tomasso, Beltrame y Lucchetti, 2011). Otro factor como practicar la propia religiosidad se correlaciona positivamente con la prestación de cuidados espirituales. El/la profesional de enfermería que cree en Dios tiende a brindar cuidados espirituales con más frecuencia y comodidad que los no creyentes (Lundmark, 2006). Asimismo, los temas relacionados con la espiritualidad/religiosidad deben de ser enseñados con intencionalidad y dirigidos hacia objetivos bien definidos. Sin estos requisitos, la educación de enfermería olvidará los contenidos de espiritualidad, como se comprobó en un estudio comparativo en Irán entre alumnos de $1 .^{\circ} \mathrm{y}$ $4 .^{\circ}$ curso de enfermería. El objetivo era comparar variables espirituales entre alumnos de distinto curso y se encontró que las puntuaciones entre ambos grupos eran similares, es decir, los alumnos de $4 .^{\circ}$ curso aun habiendo recibido 3 años más de enseñanza que los de $1 .^{\circ}$ curso, poseían los mismos conocimientos y actitudes frente al cuidado espiritual (Abbasi et al., 2014). En contraposición a estos resultados destacan los obtenidos por el grupo brasileño de trabajo de Tomasso, Beltrame y Luchetti (2011), que encontraron que los/las estudiantes de cursos más avanzados se encontraban más preparados respecto a compañeros de cursos más bajos y con opiniones acerca de espiritualidad más afines a las de su profesorado. El motivo lo asocian a una mayor experiencia clínica y mayor entrenamiento con los pacientes. Se hace evidente en todos los estudios revisados que el alumnado de enfermería, aunque difiere en el grado de motivación, carece de conocimientos básicos para brindar cuidados espirituales (Cooper y Chang, 2016); (Tiew, Creedy y Chan, 2013). Se desea que la espiritualidad sea enseñada 
como formación continua (Tiew, Creedy y Chan, 2013), como lección aparte y no de manera anecdótica en algunas asignaturas (Tirgari et al., 2013).

Por último, respecto a los beneficios de incluir materia de religiosidad/espiritualidad en los planes de estudio del grado en enfermería, se evidencia que, con diversos métodos de aprendizaje en espiritualidad y simulaciones, se obtienen numerosos beneficios. De esta forma, el alumnado tras recibir formación acerca de espiritualidad/religiosidad y cuidados espirituales se ve a sí mismo más preparado para proveer cuidados espirituales (Cooper y Chang, 2016); (Connors, Good y Gollery, 2017) y cómodo en la provisión de estos cuidados (Connors, Good y Gollery, 2017), así como más tolerantes hacia nuevas culturas y creencias, dispuesto a derivar a profesionales religiosos como capellanes hospitalarios (Cooper y Chang, 2016), con capacidad de responder de manera empática al dolor espiritual, en mayor sintonía con el paciente (Costello, AtinajaFaller y Hedberg, 2012), con conocimiento general sobre la comunicación en el cuidado espiritual (Hsiao et al., 2012), mejor manejo del patrón creencias/espiritualidad en las valoraciones de enfermería (Yilmaz y Gurler, 2014) lo que contribuye a conseguir una verdadera atención integral (Lewinson, McSherry y Kevern, 2015). A su vez se encuentran beneficios en la propia salud del estudiante como la mejora de la propia salud espiritual (Hsiao et al., 2012), mejoras en el bienestar existencial (Burkhart y Schmidt, 2012), así como el fomento de la reflexión y el alivio del estrés en las prácticas clínicas (Hsiao et al., 2012).

Aun así, se plantean desafíos para quienes están involucrados en educar a los/las estudiantes en espiritualidad. Algunos autores en pro de la enseñanza de la espiritualidad en el alumnado de enfermería investigan acerca de las mejores acciones educativas para este fin. Precisamente Croscato y Villela (2015) defienden la terapia de grupo como ocasión para adquirir habilidades en espiritualidad a la vez que contribuye a relaciones más comprensivas y en consonancia con la enfermería académica. Cooper et al. (2013) proponen invitar a los capellanes a enseñar a los estudiantes la atención espiritual desde su perspectiva. Por el contrario, Giske y Cone (2013) opinan que las competencias de los estudiantes aumentan cuando se les permite evaluar y proveer cuidados espirituales en la práctica clínica.

\section{CONCLUSIONES}

Las necesidades de formación del alumnado de enfermería en temas de espiritualidad/religiosidad varía en función del contexto sociocultural que se contemple estando ente las principales reclamaciones realizadas por el alumnado la implementación de los cuidados espirituales en la práctica clínica y la importancia a que desde el ámbito educativo universitario se enseñe a brindar estos cuidados de forma competente.

El alumnado señala numerosas dificultades a la hora de brindar los cuidados espirituales. Entre ellas, la incertidumbre y el miedo motivada por el desconocimiento en esta área; la presencia de una enfermería de orientación biológica y materialista en la práctica; la falta de profesorado adecuadamente entrenado para impartir lecciones sobre espiritualidad; y la existencia de factores personales como la falta de interés profesional y de práctica de la propia espiritualidad/religiosidad.

Tras llevar a cabo esta revisión, los 
estudios apuntan a que numerosos son los beneficios que se obtienen al incluir materia de religiosidad/espiritualidad en los planes de estudios de enfermería como que el alumnado se siente más cómodo y mejor preparado para brindar estos cuidados, con mayor manejo de la valoración integral de enfermería; y que además favorece el bienestar existencial del propio alumno.

\section{BIBLIOGRAFÍA}

- Abbasi, M., Farahani-Nia, M., Mehrdad, N., Givari, A. y Haghani, H. (2014). Nursing students' spiritual well-being, spirituality and spiritual care. Iranian journal of nursing and midwifery research, 19(3), 2427. Recuperado de http://www.ncbi.nlm.nih.gov/pubmed/24949061.

- Burkhart, L. y Schmidt, W. (2012). Measuring Effectiveness of a Spiritual Care Pedagogy in Nursing Education. Journal of Professional Nursing, 28(5), 315-321. DOI: 10.1016/J.PROFNURS.2012.03.003.

- Caldeira, S., Simões Figueiredo, A., da Conceição, A., Ermel, C., Mendes, J., Chaves, E., Campos de Carvalho, E. y Vieira, M. (2016). Spirituality in the Undergraduate Curricula of Nursing Schools in Portugal and São Paulo-Brazil. Religions. Multidisciplinary Digital Publishing Institute, 7(12), 134. DOI: 10.3390/rel7110134.

- Cone, P. H. \& Giske, T. (2013). Teaching spiritual care - a grounded theory study among undergraduate nursing educators», Journal of Clinical Nursing, 22(13-14), 1951-1960. DOI: 10.1111/j.1365-2702.2012.04203.x.

- Connors, J., Good, P. y Gollery, T. (2017). Sing Innovative Teaching Strategies to Improve Nursing Student Competence and Confidence in Providing Spiritual Care. Nurse Educator, 42(2), 62-64. DOI: 10.1097/NNE.0000000000000301.

- Cooper, K. L. \& Chang, E. (2016). Undergraduate nurse students' perspectives of spiritual care education in an Australian context, Nurse Education Today, 44, 74-78. DOI: 10.1016/J.NEDT.2016.05.020.

- Cooper, K. L., Chang, E., Sheehan, A. \& Johnson, A. (2013). The impact of spiritual care education upon preparing undergraduate nursing students to provide spiritual care, Nurse Education Today, 33(9). 1057-1061. DOI: 10.1016/J.NEDT.2012.04.005.

- Coscrato, G. \& Villela Bueno, S. M. (2015)
Spirituality and humanization according to nursing undergraduates: an action research. Investigación y educación en enfermería, 33(1), 73-82. DOI: $10.1590 / S 0120-53072015000100009$.

- Costello, M., Atinaja-Faller, J. \& Hedberg, M. (2012). The Use of Simulation to Instruct Students on the Provision of Spiritual Care, Journal of Holistic Nursing, $\quad 30(4), \quad 277-281 . \quad$ DOI: 10.1177/0898010112453330.

- Cruz, J. P., Alshammari, F., Alotaibi, K. A. y Colet, P. C. (2017) Spirituality and spiritual care perspectives among baccalaureate nursing students in Saudi Arabia: A cross-sectional study. Nurse education today, 49, 156-162. DOI: 10.1016/j.nedt.2016.11.027.

- Culatto, A. \& Summerton, C. B. (2015). Spirituality and Health Education: A National Survey of Academic Leaders UK. Journal of Religion and Health, 54(6), 2269-2275. DOI: 10.1007/s10943-0149974-4.

- Espinha, D. C. M., Camargo, S. M. de, Silva, S. P. Z., Pavelqueires, S. \& Lucchetti, G. (2013). Opinião dos estudantes de enfermagem sobre saúde, espiritualidade e religiosidade, Revista Gaúcha de Enfermagem, 34, 98-106. Recuperado de http://www.scielo.br/scielo.php?script=sci arttext \&pid=S1983-14472013000400013\&nrm=iso.

- Giske, T. (2012). How undergraduate nursing students learn to care for patients spiritually in clinical studies-a review of literature. Journal of Nursing Management, 20(8), n/a-n/a. DOI: 10.1111/jonm.12019.

- Giske, T. \& Cone, P. H. (2012). Opening up to learning spiritual care of patients: a grounded theory study of nursing students. Journal of Clinical Nursing, 21(13-14), 2006-2015. DOI: 10.1111/j.13652702.2011.04054.x.

- Hsiao, Y.C., Chiang, H.Y., Lee, H.C. \& Chen, S.-H. (2012). The Effects of a Spiritual Learning Program on Improving Spiritual Health and Clinical Practice Stress Among Nursing Students, Journal $f$ Nursing Research, 20(4), 281-290. DOI: 10.1097/jnr.0b013e318273642f.

- Kalkim, A., Midilli, T. \& Baysal, E. (2016) An Investigation of the Perceptions and Practices of Nursing Students Regarding Spirituality and Spiritual Care, Religions. Multidisciplinary Digital Publishing Institute, 7(12), 101. DOI: 10.3390/rel7080101.

- Lewinson, L. P., McSherry, W. \& Kevern, P. (2015). Spirituality in pre-registration nurse education and practice: A review of the literature, Nurse education today. 35(6), 806-14. DOI: 10.1016/j.nedt.2015.01.011.

- Lundmark, M. (2006). Attitudes to spiritual care among nursing staff in a Swedish oncology clinic. 


\section{Cultura de los Cuidados}

Journal of Clinical Nursing, 15(7), 863-874. DOI: 10.1111/j.1365-2702.2006.01189.x.

- Magalhães Oliveira, A. M., M., Lopes, M. E. L., Evangelista, C. B., Gouveia, E. M. de L., Costa, S. F. G., da \& Alves, A. M. P. de M. (2012). The spiritual dimension of care in nursing practice: student's opinion", Journal of Nursing UFPE on line, 6(9), 20372044. DOI: $10.5205 / 1981-8963-V 6 I 9 A 7306 P 2037-$ 2044-2012.

- Melguizo Herrera, E \& Alzate Posada, M. Lucía (2008). Creencias y prácticas en el cuidado de la salud. Avances en Enfermería, 26(1), 112-123. Recuperado de https://revistas.unal.edu.co/index.php/avenferm/a rticle/view/12891.

- Ministerio de Sanidad (2011). Guía de gestión de la diversidad religiosa en los centros hospitalarios. Recuperado de https://www.msssi.gob.es/profesionales/saludPubl ica/prevPromocion/promocion/desigualdadSalud/ docs/Guia_gestion_diversidad_religiosa.pdf.

- Murphy, M. M., Begley, T., Timmins, F., Neill, F. \& Sheaf, G. (2015). Spirituality and spiritual care missing concepts from core undergraduate children's nursing textbooks. International Journal of Children's Spirituality, Routledge, 20(2), 114-128. DOI: 10.1080/1364436X.2015.1055458.

- Nascimento, L. C., de Oliveira, F. C. S., Moreira Santos, T. de F., Pan, R., Flória-Santos, M., Alvarenga, W. A. \& Melo Rocha, S. M. (2016). Atenção às necessidades espirituais na prática clínica de enfermeiros. Aquichan, 16(2), 179-192. DOI: 10.5294/aqui.2016.16.2.6.

- Neil Cope, C., Azofeifa Ugalde, E. \& Calderón Montero, L. (2012). The nursing profesional's intervention with the religious needs of the hospitaliced person. Enfermería en Costa Rica, 2, 5865.

- Ross, L., Giske, T., Van Leeuwen, R., Baldacchino, D., McSherry, W., Narayanasamy, A., Jarvis, P. \& Schep-Akkerman, A. (2016). Factors contributing to student nurses'/midwives' perceived competency in spiritual care. Nurse Education Today, 36, 445-451. DOI: 10.1016/J.NEDT.2015.10.005.

- Sanabria Triana, L., Otero Ceballos, M. \& Urbina Laza, O. (2002). Los paradigmas como base del pensamiento actual en la profesión de enfermería., Escuela Nacional de Salud Pública. Recuperado de http://www.bvs.sld.cu/revistas/ems/vol16_4_02/e ms07402.htm.

- Seylani, K., Karlsson, S., Hallberg, I., Mohammadi,
E. \& Negarandeh, R. (2016). Spirituality Among Iranian Nursing Students During Undergraduate Study. Nursing and Midwifery Studies. DOI: 10.17795/nmsjournal33044.

- Tiew, L. H., Creedy, D. K. \& Chan, M. F. (2013). Student nurses' perspectives of spirituality and spiritual care. Nurse Education Today, 33(6), 574-579. DOI: 10.1016/j.nedt.2012.06.007.

- Tiew, L. H. \& Drury, V. (2012). Singapore Nursing Students' Perceptions and Attitudes About Spirituality and Spiritual Care in Practice: A Qualitative Study. Journal of holistic nursing : official journal of the American Holistic Nurses' Association, 30, 160-169.

- Timmins, F. \& Neill, F. (2013). Teaching nursing students about spiritual care-A review of the literature. Nurse Education in Practice, 13(6), 499-505. DOI: 10.1016/J.NEPR.2013.01.011.

- Tirgari, B., Iranmanesh, S., Ali Cheraghi, M. \& Arefi, A. (2013). Meaning of Spiritual Care. Holistic Nursing Practice, 27(4), 199-206. DOI: 10.1097/HNP.0b013e318294e774.

- Tomasso, C. S., Beltrame, I. L. \& Lucchetti, G. (2011). Knowledge and attitudes of nursing professors and students concerning the interface between spirituality, religiosity and health. Revista Latino-Americana de Enfermagem, 19, 1205-1213. Recuperado de http://www.scielo.br/scielo.php?script=sci arttext \&pid=S0104-11692011000500019\&nrm=iso.

- Williams, M. G., Voss, A., Vahle, B., 6 Capp, S. (2016). Clinical Nursing Education: Using the FICA Spiritual History Tool to Assess Patients' Spirituality. Nurse Educator, 41(4), E6-E9. DOI: 10.1097/NNE.0000000000000269.

- Wu, L.F., Liao, Y.C. \& Yeh, D.C. (2012). Nursing Student Perceptions of Spirituality and Spiritual Care. Journal of Nursing Research, 20(3), 219-227. DOI: $10.1097 /$ inr.0b013e318263d956.

- Yilmaz, M. \& Gurler, H. (2014). The efficacy of integrating spirituality into undergraduate nursing curricula, Nursing Ethics, 21(8), 929-945. DOI: 10.1177/0969733014521096.

- Zakaria Kiaei, M., Salehi, A., Moosazadeh Nasrabadi, A., Whitehead, D., Azmal, M., Kalhor, R. \& Shah Bahrami, E. (2015). Spirituality and spiritual care in Iran: nurses' perceptions and barriers. International Nursing Review, 62(4), 584592. DOI: $10.1111 /$ inr.12222. 